extreme depression, physical and moral. He was kept quiet, took bromide of potassium and a large amount of nutrient fluids; a blister also was applied behind the left ear. The deafness and pain in the mastoid region were relieved, and be slept a little better, but a deep-seated sense of pain came on in the right side of the head, and a few days later severe frontal and orbital neuralgia, with intense congestion of the conjunctiva of the left eye. He had at the same time severe urticaria, which was no doubt an expression of nerve disturbance. The hemiplegia remained the same. Large doses of quinine were now given, and with benefit, but no impression was made on the disease until a distinct syphilitic manifestation was discovered, and iodide of potassium was given, when he rapidly recovered.

Syphititic inflammation of dura mater.-Alfred $\mathrm{P}-$, aged thirty-one, a sbopman, was in St. Mary's Hospital under my care from Oct. 2nd to Nov. 25th, 1872, complaining chiefly of great pain in the head. He had contracted syphilis ten years before; had had slight and transient cutaneous manifestations, and nothing further till within fifteen months. He had begun to suffer from nodes and a tubercular eruption, for which he had been treated by Mr. Lane for nine months, talking iodide of potassium in doses of ten grains, with latterly fifteen or twenty grains of the bromide. For three weeks he had had severe nocturnal pains in the head, attended with slight delirium at times. His appetite was bad; bowels confined; no vomiting; pulse 84 ; temperature $98^{\circ}$. I gave him eighteen grains of the iodide three times a dav, with carbonate of ammonia and tincture of quinine, and chloral at night. On Oct. 8 th be was no better, and chloral in large doses and morphia injections had failed to give him sleep. The dose of the iodide of potassium was therefore increased to twenty-four grains, and leeches were ordered to each temple, and next night one behind each ear. The leeches applied to the temples afforded relief, not those bebind the ears, and on the 13th and 15th others were applied. The iodide was now again raised to thirtysix grains three times a day. On the 17 th he appeared better, had much less headache, and was eating well ; complained of thirst. Urine abundant, specific gravity 1125, no albumen; pulse and temperature normal. Two days later, however, the headache had returned, and he had pains in the limbs; a tubercular eruption also had appeared on the nose and face. I now gave him the one-twelfth of a grain of biniodide of mercury with quinine in the form of a pill twice a day, reducing the dose of iodide of potassium to eighteen grains three times a day. He continued, however, to get worse, and, in addition to the pain in the head, he complained of soreness in all the limbs; his behaviour was strange, and his mind evidently affected. On the 27 th leeches were again ordered; but the relief given was slight, and erysipelas came on round the punctures. The medicines were consequently suspended, and the erysipelas was treated with local applications. On the 30 th the inflammation had subsided, but the tuhapalar eruption had increased on the forehead and nose, and apneared on other parts; the headache and bone pains had returned in a severe form; there was slight loss of power in the left arm, and the mental condition was peculiar. He was perverse and unreasonable, very strange in his temper, and one day at my visit he produced his dinner, which he had concealed for the purpose. I now resumed the administration of the biniodide of mercury and iodide of potrssium; the latter in doses of twentyfour grains. He renained in much the same condition for a week, at the end of which time the gums were affected. With this the headache had gone, the mental oppression had cleared off, and in a short time the eruption faded and disappeared. The mercury was discontinned, and the iodide of potassium reduced to sixteen grains. He was discharged, feeling well, on Nor. 25th. Since then he has been under treatment again and again, and twice has required the administration of mercury. While taking the iodide of potassium in large doses-eighteen to twenty-four grains-be lrept well; but once I allowed him to leave it off; on two or three occasions he neglected to attend at the hospital ; and once it was found that the prescribed amount of iodide was not given. On each occasion a relapse occurred, and the iodide was not always found sufficient to give relief; but recourse was had to biniodide of mercury, and occasionally a leech was applied.

In November, 1872, I saw with Dr. Langmore a patient who was the wife of a man who at the time had severe syphilitic rupia. She had had one miscarriage, and no living children; no syphilitic manifestations. Six months before I saw her she had had paralysis of the right side of the face, and numbness in the right arm, which recovered in six weeks. She had been complaining of numbness round the mouth and in the left arm, nnd she suffered from intense frontal headaches, which generally came on at 3 A.M. Sensation was found to be impaired in the left arm, and the grasp was weak. She walked well. Sensation was also.deficient over the lower part of the right side of the face in the area of distribution of the two lower divisions of the fifth, the anosthesia transgressing the median line about the mouth. The forehead, the tip of the nose, and the tongue were not affected. The sight was not good, and she was unable to read long; the optic discs were pink, striated by vessels, and the retinal veins were large, but there was no appearance of swelling, and the dises were well defined. The patient was taking iodide of potassium in doses of six grains, and it was agreed that the dose was to be gradually increased to twenty-four grains. This failing, mercury was to be tried.

Here the cross paralysis, incomplete anæsthesia in the two lower divisions of the right fifth nerve, and weakness and impaired sensation in the left arm, pointed to a lesion near the apex of the petrous bone, or the adjacent part of the sphenoid, involving the two branches of the trigeminus after their departure from the Gasserian ganglion and the crus cerebri. Had the entire fifth nerve been affected, the seat of disease might have been the pons. The severe pain led me to conclude that the disease had its starting point in the bone or dura mater, and I anticipated difficulty in affording relief. I learn, however, that she improved greatly under the iodide of potassium. She is liable to headaches, but there is now no paralysis.

\section{OPHTHALMOSCOPIC EXAMINATION DURING AN ATTACK OF EPILEPTIFORM AMAUROSIS.}

BY J. HUGHLINGS JACKSON, M.D., F.R.C.P., PHYSICYAN TO THE LONDON HOSPITAL AND TO THE HOSPITLL FOE THE FPILEPTIC AND PARALYSED.

THIs patient had attacks of what $I$ used about ten years ago to call epilepsy of the retina, but which $I$ have for some time called epileptiform amaurosis. It is the most striking case of the kind I have seen. The most common clinical association of paroxysmal defects of sight is convulsion beginning unilaterally, as I have long noticed. There was no such association in this case. The phenomena are sometimes positive-e. g., colours, sparks, \&c.; in other cases, as in the following case, negative. Another peculiarity of this case is that there was neuritis, and that too with considerable impairment of sight.

Henry G-, aged thirty-four, was an in-prtient under my care June 27 th, 1870 , for symptoms which I believed to depend on intra-cranial tumour, certainly on severe encephalic disease. Very careful notes were taken of his case by my colleague, Dr. Gowers, but I extract from them only so much as bears on one point-namely, paroxysmal loss of sight. I first give what my colleague gathered from the patient as to this phenomenon.

"June 16th.-During the last two or three days he has had occasional attacks of loss of sight coming on gradually and lasting several minutes-he says, five or ten ; there is not complete darkness, but a degree sufficient to prevent him from distinguishing any object. Headache, frontal, and aching in the limbs accompany the attacks; the headache continues afterwards."

It is to be noted that at this time be could read No. $1 \frac{1}{2}$ Snellen with each eye. There was, however, double optic neuritis. I have for years insisted on the fact that sight may be good when the optic dises are very bad. He continued subject to the above described paroxysms, and by July lst his sight had failed so that he could at no time read No. 20 of Snellen. Now comes the part to which I wish particularly to draw attention. Mr. Burgess had made me a drawing of the fundus of one of the patient's ejes, and I went to the hospital to compare it with the 
"specimen" itself. The following is an account of what took place.

July 4th.-This morning, when I was speaking to him, he said nervously, "The blindness is coming on." We put him in a chair; he said he could see nothing. To test this I put my fingers before his eyes, jobbing them forwards, as if I would put them into his eyes; this produced no effect until I touched the lashes. In about half a minute he said be could see a "light object," but further improvement was slow and the blinduess returned. I took him, in this second attack, to the gas-lamp; he "thought he saw a light" when I threw the light into his eye for ophthalmoscopic examination. I discovered no change of importance. As just before I had been comparing his left disc with Burgess's drawing, a notable difference could not have escaped me. The large veins in the left eye were, perhaps, darker than before the paroxysm, an observation of no definiteness. It was curious to note the difference in his "ocular manner" from that during a former examination. From habit, I kept saying, "Lor k at my little finger"; and now, he being temporarily blind, no result followed. After writing down the latter part of the above I returned to him. He then said he could see objects, and he counted my fingers and followed quickly and very correctly the movements of $\mathrm{my}$ hand-that is, he directed his eye to the finger I asked him to look at. At that examination-i. e., after the second paroxysm instanced -I felt sure that the veins (I speak only of the left) were not as before the first attack; they were not so dark, and seemed as if slightly collapsed, being, to use an exaggerated expression, beaded. A few minutes later he could puzzle out No. 20 of Snellen.

Under treatment by large doses of the iodide of potassium he improved, and on Ang. 1st left the hospital feeling quite well. He could read No. 1. However, he soon got worse again, and had a severe illness, with palsies of ocular nerves, \&c. He recovered from these symptoms too, except that he remained blind. He is still suffering, having occasionally pain in the head; but his general health is good. Manchester-square.

\section{A CASE OF RETENTION OF URINE FROM IMPASSABLE STRICTURE,}

RELIEVED BY ICE IN THE RECTUM, AND THE STRICTURE SUCCESSFULLY TREATED BY CAUSTIC POTASH.

BY W. F. TEEVAN, B.A., F.R.C.S.,

SURGEON TO THE WEST LONDON AND ST. PETER'S HOSPITALS; LATR LRCTURER ON ANATOMY AT THE WRSTMINSTER HOSPITAL.

For all practical purposes, strictures of the urethra may be divided into two kinds, the Passable and the Impassable; and the former are as easy to treat as the latter are difficult. An attempt to relieve retention of urine from an impassable stricture is not always successful, and an operation has sometimes to be resorted to. Any remedy, therefore, for this complaint which is harmless in its operation and speedy in its effects commands our attention. I believe M. Cazenave, of Bordeaux, was the first to introduce into practice, some quarter of a century ago, the plan of treating retention of urine by plugging the rectum with ice; and if the method is only half as successful as he claims it to be it will prove a valuable auxiliary to the surgeon. When called to a case of retention, whatever may be its cause, I always endeavour at once to pass an instrument; and I only resort to other means when foiled with the catheter. In the case I am about to relate $I$ had in vain endeavoured to introduce different instruments, and, knowing how highly M. Cazenave had extolled the merits of ice in similar instances, I determined to give his recommendation a fair trial; with what result will be seen.

On October $18 \mathrm{th}$, J. B-, a labourer, aged forty-six, came under my care at St. Peter's Hospital as out-patient, for an impassable stricture of long continuance, situated five and three-quarter inches from the meatus externus. Immediately in front of the stricture, in the median line, there was a false passage into which a catheter could be passed up to the hilt without drawing a drop of blood; thus demonstrating that it had acquired the respectability of age. On October 18tb, and again on the $20 \mathrm{th}$, I tried in vain to introduce different instruments; and on the $22 \mathrm{nd}$ the patient, came to the hospital with an attack of retention of urine. Mr. Walter Coulson, who bappened to be in the institution, endeavoured, without success, to pass a catheter, and I was sent for to take charge of my case. On my arrival I at once employed the catheter, but without any result; and therefore resorted to M. Cazenave's plan, and plugged the rectum with small pieces of ice. Exactly twenty minutes after the last fragment had been inserted into the bowel the patient began to pass water guttatim, and in the course of half an hour contrived to empty his bladder. He was kept quiet and warm in bed, and his bowels were gently moved every day. I endeavoured every other day, for about five minntes, to vass an instrument, but without success and on Nov. 1lth $I$ had his perineum freely leeched, and continued my attempts at catheterism, with no better result till Nov. 24,th, when I fancied I had succeeded in introducing a very slender whalebone bougie into the bladder. In order to demonstrate the accuracy of my supposition, and to ntilise its realisation, if correct, I attempted to slide one of $m y$ smallest grooved silver catheters over the bougie into the bladder; but in doing this I used an amount of force which nothing but the intractable and serious nature of the complaint could justify, and the result was that the bougie was cut in two about its centre, six inches of the whalebone being left behind in the stricture. As the patient experienced no pain from its presence, I determined to leave it in the urethra, in the hope that it would set up a profuse dis. charge, and so facilitate the treatment. In this expectation I was, however, disappointed; and on Dec. 1st I removed the broken portion of the bougie after its seven days' imprisonment, the patient being all the while entirely ignorant of its whereabouts, and complaining of notbing beyond an occasional smarting when passing water. I now directed the patient to pass down a large bougie as far as it would go, and to keep it pressing against the face of the stricture for half an hour every day. Twice a week I tested the nrethra with instruments to see if any progress were made, but the results were always of a negative character. The man's stricture possessed but little sensibility, and bence the un. remitting assaults made on it were productive of neither pain nor annoyance.

On Dec. 19th I came to the conclusion that a fair and unusually patient trial had been afforded for the success of all ordinary means, and nothing apparently remained but the knife to cut the Gordian knot. As, however, the patient's perineum was intact, I was loth to cut into it, and the presence of a false passage did not render the most difficult operation in surgery more easy of performance. On reflection, I considered that this was a fit and proper case on which to try the "bougie armée," and I communicated my intention to Mr. Henry Smith, the redoubtable champion of this potent machine, who was kind enough to intimate to me his gladness to be present when I used it. Accordingly, on Dec. 22nd, in the presence of Mr. Smith and two other gentlemen, I passed down to the stricture a wax bougie, slightly curved, and armed with a small piece of caustic potash. For a couple of minutes I kept the bougie firmly pressed against the stricture, slightly rotating it whilst doing so; and on withdrawing it, I found the potash had all deliquesced against the stricture. Being aware of the exact relations of the false passage, I was particular in keeping the bougie's point pressed slightly upwards, in order to avoid entering the mouth of the abnormal route in the lower wall. The application of the caustic was not attended with any pain or bleeding, and on Dec. 25th I reapplied it, the patient complaining that the orifice was very sore, as its lips had tasted the caustic on its entry to the urethra.* The following day the man stated that his stream of water had much improved, but there was a gonorrboal discbarge, with, however, diminished frequency of micturition. On Dec. 27 th I again employed the "bougie armée" as before; and on the 31st, knowing the instrument had penetrated about a quarter of an inch deeper than on the first occasion, I determined to endeavour to pass a very small olivary bougie. Accordingly, before using the bougie, I gave it the well-known coudée turn of M. Auguste MIercier

* When I next require to use the " bougie armé" I shall apply it through the urethral tube used for the endoscope. 